\title{
Millisecond structural dynamics during the piezoelectric cycle of silk fibroin by synchrotron X- ray scattering \& comparison with DFT calculation
}

\author{
C. J. Garvey ${ }^{1,2}$, S. T. Mudie ${ }^{3}$, D. Music ${ }^{4}$, P. A.T. Olsson ${ }^{4,5}$, V. Sencadas ${ }^{6}$
}

${ }^{1}$ Lund Institute for Advanced Neutron and X-ray Science, Lund 20503, Sweden, ${ }^{2}$ Heinz Maier-Leibnitz Zentrum (MLZ), Lichtenbergstrasse 1 85747, Garching, Germany, ${ }^{3}$ Australian Synchrotron, ANSTO, 800 Blackburn Rd, Clayton, 3168, Australia, ${ }^{4}$ Department of Materials Science and Applied Mathematics, Malmö University, SE-205 06 Malmö, Sweden, ${ }^{5}$ Division of Mechanics, Lund University, Box 118, 22100 Lund, Sweden, ${ }^{6}$ School of Mechanical, Materials, Mechatronic and Biomedical Engineering, University of Wollongong, Wollongong, NSW, 2522, Australia

Christopher.garvey@tum.de

While simple associations exist between piezoelectric properties and processing history, there is considerable scope for design of materials based on a more detailed molecular understanding of the re-arrangements that underpin the piezoelectric phenomenon in silk fibroin.[1] Crystallinity, and the two-phase model of semi-crystalline model of polymers, are often used to understand the properties of protein based materials where there is considerable thermodynamic drive to short range ordering of polymer chains, folding, which is not present in melt processed thermoplastics. Our investigations aim to probe the relationship between structure and dynamics in silk fibroin based materials and correlate these with the piezo-electric signal.

Recently, we have used a triggered and summative data acquisition scheme to synchronise X-ray scattering data collection with a piezoelectric cycle of a compressed electro-spun fibroin mat.[2] This mode enabled a steady perturbed state to be sampled. The summation of the scattering patten from this state across multiple cycles provides superior statistics than could be achieved by sampling a single cycle. The setup is shown in Figure 1A. At rest this poorly ordered system exhibits a limited number of very broad peaks but quite a high degree of chain folding.[3] With compression there is marked increase in the scattered intensity, both in the small (SAXS) and the wide (WAXS) angle regimes, as well as a shift and reduction in broadness of the WAXS peaks (Figure 1B). We interpret the increase in the SAXS signal as an increase in scattering from grain boundaries and the WAXS as the formation of new crystalline domains. However, the limited number of very broad diffraction peaks make these data unsuitable for structural determination.
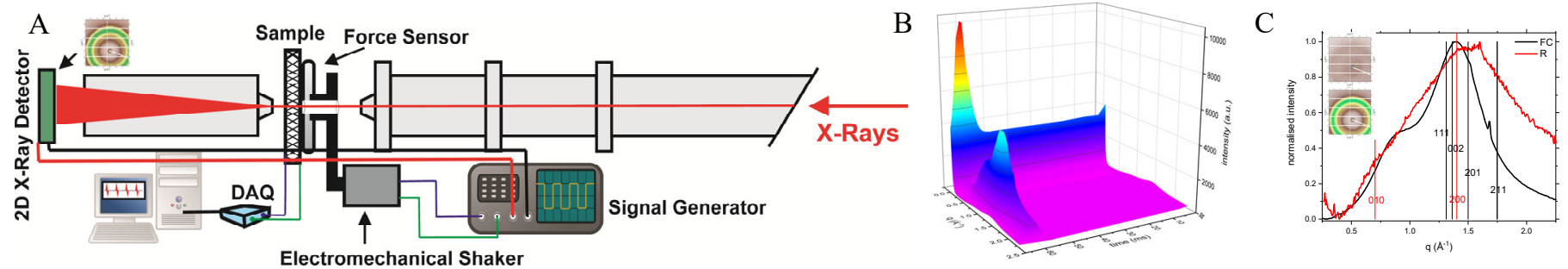

Figure 1. A. Experimental set-up for the acquisition of time resolved X-ray diffraction during the piezo cycle. Adapted from ref [2]. B. 1D profiles during the piezoelectric cycle. C. Peak normalized intensity for fully relaxed (red line - indexing red vertical lines); and peak normalized intensity for fully compressed (black line) and the black vertical lines represent based on DFT calculations.

In order to provide an alternative, but complementary, perspective on the structural dynamics and the nature of the potential surface along which the polymer folds, during the piezo cycle we have turned to a computation approach. Density functional theory (DFT) calculations were performed within the framework of the projector augmented wave potentials parametrised by Perdew et al., [3] and the Tkatchenko-Scheffler correction [4] with a self-consistent screening to account for the weak correlations. Full structural optimisation of orthorhombic $\mathrm{C}_{20} \mathrm{O}_{8} \mathrm{~N}_{8} \mathrm{H}_{32}$ was performed. The calculated lattice parameters $(a=9.409 \AA, b=6.984 \AA, c=9.221 \AA)$ and the corresponding diffraction pattern (black vertical lines) are compared with the experimental data in Figure 1C.

[1] Rockwood, D. N., Preda, R. C., Yücel, T., Wang, X., Lovett, M. L. \& Kaplan, D. L. (2011). Nature Protocols 6, 1612.

[2] Sencadas, V., Garvey, C., Mudie, S., Kirkensgaard, J. J. K., Gouadec, G. \& Hauser, S. (2019). Nano Energy 66, 104106.

[3] Perdew, J. P., Burke, K. \& Ernzerhof, M. (1996). Physical Review Letters 77, 3865-3868.

[4] Tkatchenko, A. \& Scheffler, M. (2009). Physical Review Letters 102, 073005.

\section{Keywords: piezoelectricity; biopolymer; synchrotron wide angle X-ray scattering; millisecond resolved diffraction}

The authors acknowledge beamtime on the SAXS/WAXS beamline at the Australian Synchrotron, part of ANSTO. The computations were performed on resources provided by the Swedish National Infrastructure for Computing (SNIC) at National Supercomputer Centre (NSC) in Linköping, Sweden. CJG acknowledges the support of a LINXS fellowship.

Acta Cryst. (2021), A77, C368 